\title{
Cellular therapy for traumatic neurological injury
}

\author{
Charles S. Cox Jr ${ }^{1}$
}

Neurological injury is the primary lethal mechanism of injury in children, and the primary etiology of long-term disability after trauma. Laboratories and clinical/translational teams have sought to develop stem/progenitor cell therapies to improve recovery in a clinical setting in which there is no significant reparative option. While none of these treatments are currently standard therapeutics, phase Ilb clinical trials are underway in both adults and children in severe traumatic brain injury (TBI) and phase I/Ila trials in spinal cord injury. This review will characterize the cell therapy strategies: cell replacement and tissue integration vs. immunomodulation/ enhanced endogenous tissue repair. TBI is somewhat different from other central nervous system injuries (spinal cord injury and stroke), in that TBI is a diffuse injury, whereas spinal cord injury and stroke are anatomically discrete. Importantly, this drives cell therapy approaches, as TBI is less apt to be treatable with a local cell replacement intervention. More localized injuries may be more amenable to local approaches and cell replacement to bridge focal gaps. This review focuses on a few reports in the field that highlight areas of progress, but is not intended to be a comprehensive survey of the state of regenerative medicine for neurological injuries.

\section{BACKGROUND}

\section{Traumatic Brain Injury}

A pproximately 1.5 million people $(40,000$ pediatric emergency department visits, 30,000 pediatric hospitalizations, and 3,000 pediatric deaths) suffer traumatic brain injury (TBI) yearly in the United States. The annual mortality approaches 50,000 with the remaining patients suffering from varying levels of long-term impairment. Overall, 6.5 million patients are burdened by the physical, cognitive, and psychosocial deficits associated with TBI, leading to a total economic impact of $\sim 60$ billion dollars (1). There is currently no effective pharmacologic therapeutic for TBI (2). Since the 1970s, TBI has been described as "primary injury" and "secondary injury." The primary injury is the immediate result of energy dissipation within the substance of the brain, and occurs within milliseconds of impact. Primary injuries include direct neuronal, glial, and vascular disruption. Secondary injuries are the result of the reactive biochemical events that occur after the primary injury. These reactive biochemical events may accelerate or exacerbate initial cellular injury or cause "new" injury $(3,4)$. Current TBI management strategies are designed to minimize secondary injury and their consequences (5-7). The current treatment paradigm focuses on the evacuation of extra-axial fluid collection(s), and the management of cerebral hemodynamics by reducing intracranial pressure (ICP) or increasing mean arterial pressure (MAP) to optimize cerebral perfusion pressure (CPP) $(8,9)$. There are currently no proven treatments to prevent secondary brain injury after TBI, which can be manifest acutely as elevated intracranial pressure as a consequence of increased blood-brain barrier (BBB) permeability and cerebral edema. Elevated ICP has been correlated with poor clinical outcomes. Clinically, secondary brain injury associated with TBI is associated with neuroinflammation and the subsequent cerebral edema, leading to an increase in ICP and a subsequent decrease in cerebral perfusion. If poorly controlled, elevated ICP will impair cerebral perfusion and exacerbate the primary injury. Significant additional advances in reducing the disability associated with TBI are unlikely using the current management strategies alone (2). For pediatric patients suffering a severe TBI, there is an $\sim 30 \%$ chance of a bad outcome-defined as death, vegetative state, or severe disability (10).

\section{Spinal Cord Injury}

Spinal cord injury (SCI) continues to be a significant cause of disability despite extensive research and supportive clinical care. Similar to TBI, no reparative therapies exist for SCI. There are $\sim 11,000-20,000$ new cases/year $(\sim 1,500$ pediatric) in the United States, and 250,000 patients live with SCI. SCI consists of both the kinetic primary injury and the biochemical secondary injury, characterized by inflammation, cytotoxic depolarizations, which can lead to further neuronal loss, and oligodendrocyte apoptosis at a distance from the primary lesion. The loss of oligodendrocytes results in myelin loss and subsequent poor action potential conduction. This pathophysiology is the foundational concept of neural stem cell (NSC)/oligodendrocyte precursor cell (OPC) replacement strategies to restore function after SCI.

\section{Overview of Cell Therapy for Neurological Injury}

Over the past 10 years there has been a growing body of literature supporting the use of various progenitor cell types 


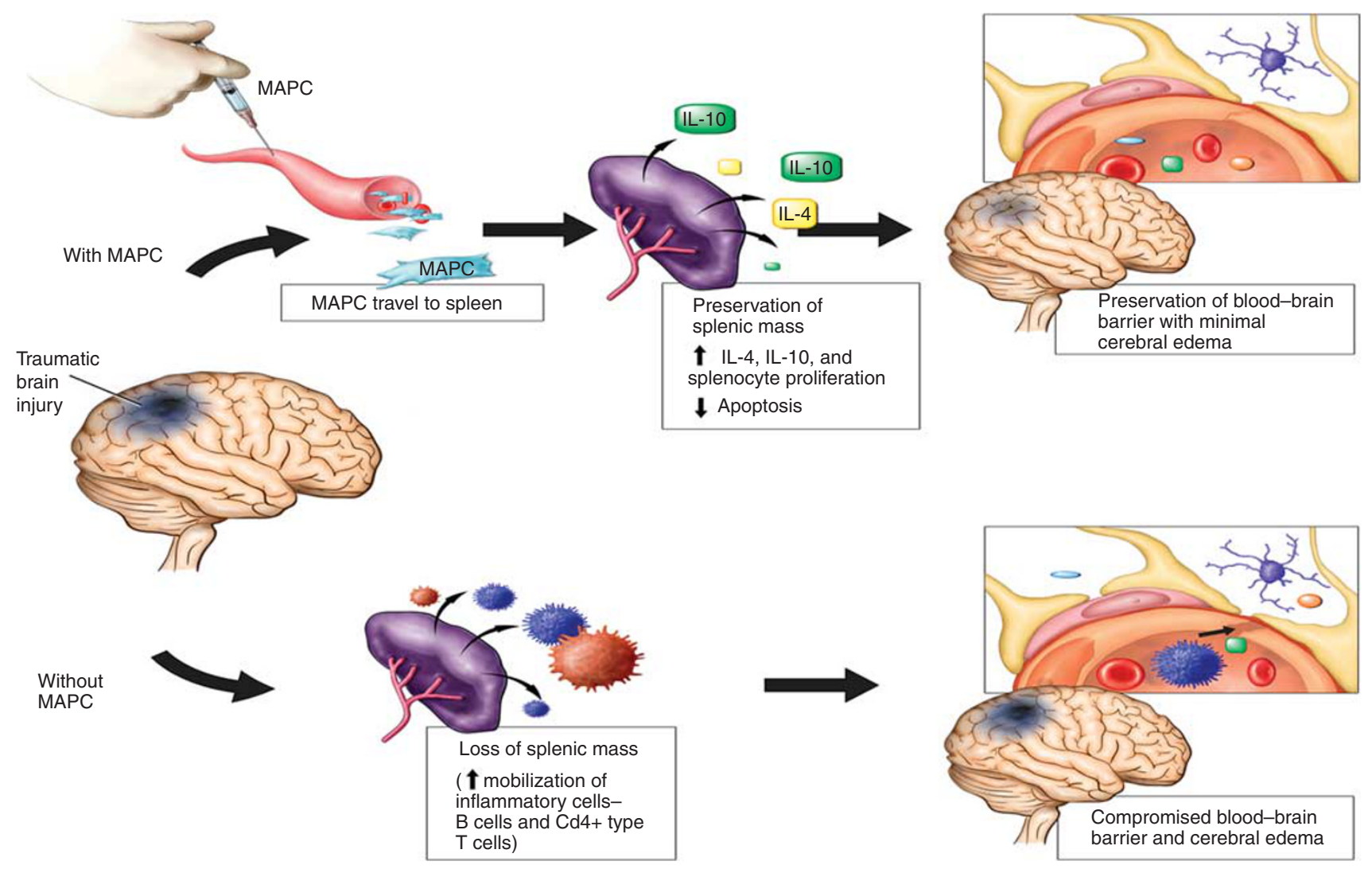

Figure 1. Systemically injected multipotent adult progenitor cell (MAPC) interact with splenocytes to exert anti-inflammatory effect. This cartoon demonstrates the concept of peripheral immune interactions that influence central nervous system (CNS) inflammatory responses to injury. Through cell-labeling experiments, we demonstrated that these cells sequester in the spleen and lung, resulting in an efflux in anti-inflammatory cytokines, and T-regulatory cells. This correlated with polarization towards an M2 predominant microglial phenotype and functional improvements. This figure was adapted from our original publication in Experimental Neurology (16).

to treat acute neurological injuries such as TBI and stroke. NSCs (adult and embryonic), mesenchymal stromal (MSCs) and multipotent adult progenitor cells (MAPCs), and bone marrow mononuclear cells (from which MSC and MAPCs are derived) have all shown efficacy in preclinical models of TBI/ stroke through various mechanisms; however, few groups believe that true neural replacement and integration are the mechanisms involved in the observed efficacy. More likely is that the progenitor cell populations are modifying the regional response to injury (inflammatory/reparative vs. regenerative), resulting in improved functional outcomes.

Bone marrow-derived adherent/expanded cells: MSCs (auto and allogeneic \pm modified) and MAPCs. MSCs are a similar cell type relative to MAPC, in that they are an adherent, bone-marrow-derived cell, but they differ in terms of ultimate product profile in terms of potency, size, and expansion characteristics. There are numerous publications that report the efficacy of MSCs in animal models of TBI, and this was reviewed in a meta-analysis of preclinical models of TBI using a cellular therapeutic (11). A reported mechanism of action is that cells migrate to the site of injury and exert a paracrine effect by secreting growth factors that preserve at risk neurons. We could not reproduce those data nor could we confirm reports of cell engraftment (12). Our findings of lack of MSC survival/engraftment in the central nervous system (CNS) have been confirmed by others (13). Finally, we noted that the degree of growth factor production and MSC distribution within the CNS as reported could not produce a clinically meaningful concentration of the putative mediators of the observed improvements (14). Preclinical data using MAPC in a rodent model of TBI demonstrated improved functional outcome at 120 days after TBI, as well as preservation of the blood-brain barrier in the acute phase of injury (15). Mechanistically, these cells upregulate the antiinflammatory response to injury via interactions with splenocytes (16). Ultimately, there is an alteration in the microglial activation status to a reparative vs. inflammatory phenotype (14). (Figures 1 and 2) All of these data support the working hypothesis that MAPC infusion produces the observed functional benefit by altering the innate immune response to TBI (secondary brain injury), and that engraftment is neither required nor desired for the effect to occur.

Nonexpanded/noncultured bone marrow mononuclear cells contain MSCs/MAPCs in small numbers as well as a host of hematopoietic progenitors and monocytes/lymphocytes. These bone marrow mononuclear cells have similar characteristics to umbilical cord blood mononuclear cells and both 


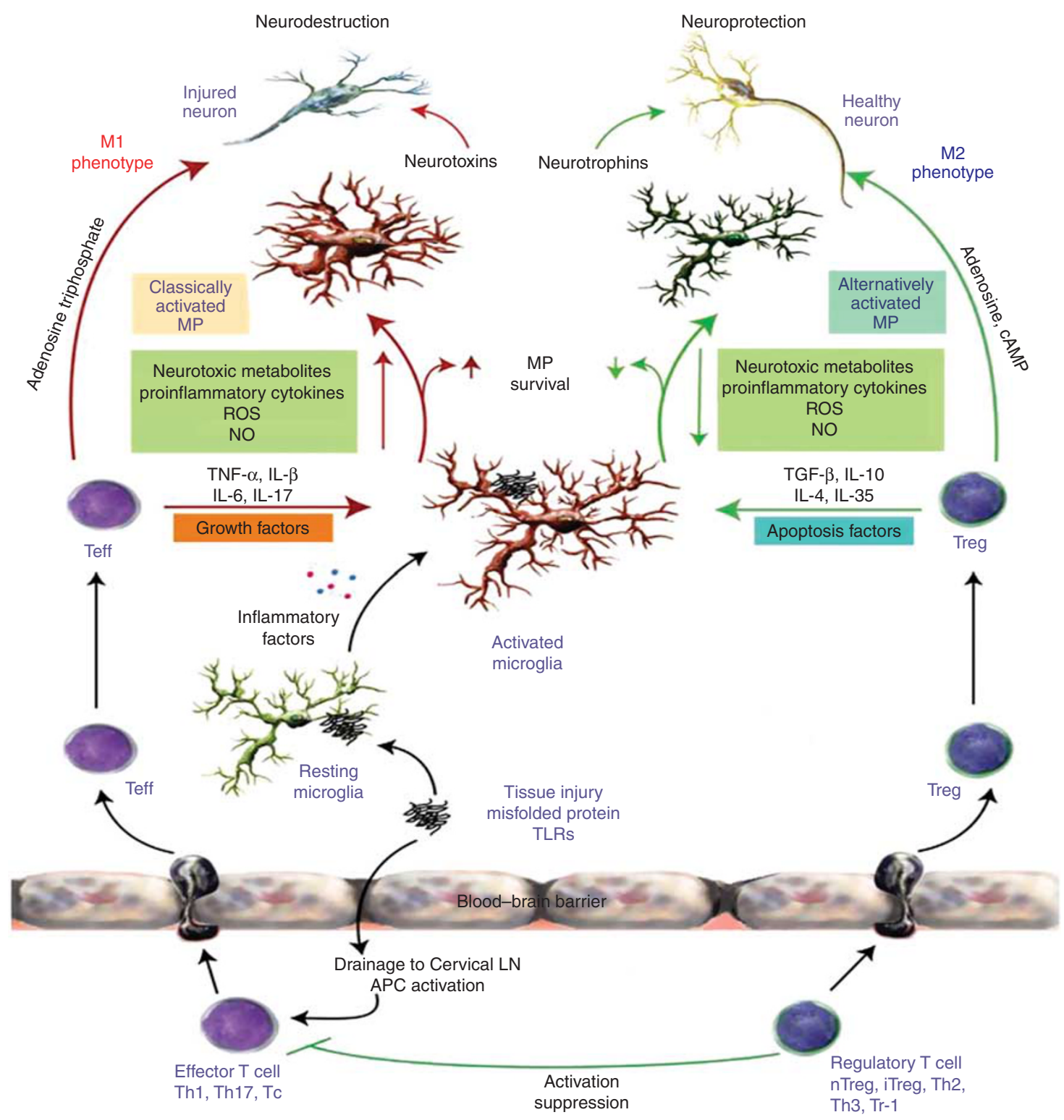

Figure 2. Cellular therapies polarize the microglia to an anti-inflammatory M2 phenotype via pleiotropic mechanisms of action. Microglial polarization is important as these are the primary effector cells of the neuroinflammatory response to injury.

cell populations have proven efficacious in proof of concept studies treating TBI $(15,17)$. Similar work, with parallel results, have been done in stroke with both cell types (18-20).

ESC-derived NSC or oligodendrocyte stem cell/OPC. This paradigm uses a starting bank of embryonic stem cell (ESC) to expand a large number of cells, and then they are differentiated in culture to the proposed neural precursor cell. The use of NSCs or OPCs to replace damaged neurons and supporting cells is conceptually attractive, but difficult due to a host of practical issues and barriers. Primarily, TBI is a multifocal disease, thus targeted delivery of a cell product or even a cell/scaffold composite is hampered by the numerous areas of injury. While there may be a dominant contusion visible on initial imaging, this may not be the primary lesion of clinical significance. These cells do not migrate in the adult brain to any clinically relevant distance, so this requires multiple, stereotactic injections. In the clinical setting of diffuse injury/multiple lesions, it can be difficult to discern the specific lesion responsible for the clinically dominant pathology. This is easy to model in animal experiments, but difficult to translate into heterogeneous contusion/diffuse axonal injury patterns that occur in patients. Secondarily, the issue of immunosuppression to ensure engraftment is potentially problematic in a trauma population, but has proven feasible in the chronic patient. Finally, the complication of ectopic tissue development can be especially problematic in the CNS as opposed to many other potential sites. This concern is real, but probably overstated with current approaches for cell selection. However, the concern is that even a very small number of residual nondifferentiated ESCs 
(in theory only one is required) can result in teratoma development (21). These barriers will make this approach a more distant possibility, and even if successful, could be complementary to systemic, immunomodulatory approaches.

Cell-derived proteins. Supernatants or conditioned media have been used successfully in preclinical models of TBI with striking success. In a mouse model of TBI, we have shown an effect on BBB permeability, and enhancement of neurogenesis due to TIMP-3 (tissue inhibitor of metalloproteinases-3) production by MSCs (22). Similarly, Prockop's and coworkers (23) has shown that TSG-6 production from MSCs produces similar early neuroprotective effects and late functional behavioral improvement in mice. One of the primary barriers in translation of using the protein alone is the scale-up to produce the proteins in a clinically relevant volume (DJ Prockop, personal communication). While this is ultimately a solvable problem, it could make the cost of this therapy beyond the reach of any practical application. The use of exosome-derived proteins from stimulated MSCs or other adherent, bone marrow-derived cell types may also be a useful, next-generation approach. Zhang et al. (24) have shown that MSC-derived exosomes improve functional recovery in rodent $\mathrm{TBI}$, and the putative mechanism is via transfer of specific miRNAs. Limitations of this approach hinge on the identification of the most effective molecule/ protein, while cell-based therapies affect multiple mechanisms of action for an overall pleiotropic effect.

\section{PRECLINICAL DATA}

\section{Traumatic Brain Injury}

There is extensive preclinical data studying stem cell-based therapies in models of TBI. Conceptually, the field started in the 1990s with the concept of neural replacement with claims of transdifferentiation of MSCs into neurons when directly implanted into the brain. This was later disproven, and it is now well established that MSCs and similar cell types do not engraft in any relevant amount (13). Of the systemically infused cell types used to treat TBI (umbilical cord blood mononuclear cells, MSCs, MAPCs, bone marrow-derived mononuclear cells, adipose- and placental tissue-derived MSCs, and others), there are striking similarities in the functional outcome improvements (15,25-28). Similar results have been obtained with direct perilesional injections of these cell types as well as various NSCs (29). These commonalities demonstrate that there are probable conserved, pleiotropic mechanisms of action of various cell populations after injury. It is less likely that each cell type has a unique mechanism of action to produce similar functional improvements. The field has come to agree that the likely multiple actions are related to the cell secretome, infused cell-innate immune cell interactions that ultimately serve to polarize the microgila/ infiltrating macrophage populations to a reparative vs. inflammatory phenotype, and a downregulation of proinflammatory cytokine signaling. Engraftment/replacement has not been demonstrated.

\section{Spinal Cord Injury}

A number of cell therapy approaches have been explored as a therapeutic strategy for SCI repair. In broad terms, these can be classified as neural replacement or supporting cell replacement to augment neural repair/salvage. A NSC strategy (with differentiation into neurons, as well as supporting cells such as astrocytes, oligodendrocytes, and other glial elements) using NSCs derived from ESCs or fetal subventricular zone NSCs directly implanted into the perilesional area of the injury was developed in various laboratories. A different conceptual approach using an ESC-derived OPC was pursued by the Steward laboratory (30), Cao et al. (31), and others. The OPC approach is appealing owing to the demyelination away from the central lesioned area. The preclinical data demonstrate that when OPCs are directly implanted into the lesioned spinal cord, they can remyelinate damaged (but viable) neurons and serve to improve locomotion. While many in the field have made substantial progress in this area, two labs developed the large portfolio of data that allowed the NSC and OPC approaches to be translated into clinical trials $(28,32)$. Both groups did exhaustive safety, proof of concept, scale-up, confirmatory efficacy and dose ranging studies that were compelling enough to allow a relatively high risk procedure to move forward (cervical spinal cord direct injection of stem cells).

\section{EARLY CLINICAL TRIALS}

\section{Traumatic Brain Injury}

Pediatric phase I BMMNCs. One of the first cell therapy trials for TBI was completed in children, but not adults (33). The reason for this is the leading cause of trauma-related deaths is severe TBI. Further, there are no reparative therapies, so the barrier to developing any potential therapeutic is lower. Three major findings were noted in this trial: (i) acute bone marrow harvest/infusion in the acute injury setting is safe and logistically feasible, (ii) there was none of the expected postTBI progressive CNS tissue loss (8-12\%) with cell therapy $(34,35)$, and (iii) there was progressive improvement in clinical outcome after 6 months (Figures 3 and 4).

PILOT: After completion of the phase I pediatric clinical trial and the observations in preclinical studies that bone marrow mononuclear cells (BMMNCS) may reduce $\mathrm{BBB}$ permeability and the neuroinflammatory response to TBI, our group retrospectively evaluated the therapeutic intensity level (PILOT score) required to treat elevated intracranial pressure (36). This served as a surrogate for inflammation-associated cerebral edema and $\mathrm{BBB}$ dysfunction. Case- and timematched controls were compared WITH the treatment group that was reported in the initial trial. Recognizing all of the limitations of post hoc analysis and retrospective data collection, there was a clear reduction in therapeutic intensity in patients treated with autologous BMMNC relative to controls. Further, there was a reduction in the average maximum daily ICP burden, which has been definitively correlated negatively with outcomes (37). Importantly, these data supported the preclinical findings that were the proof of 


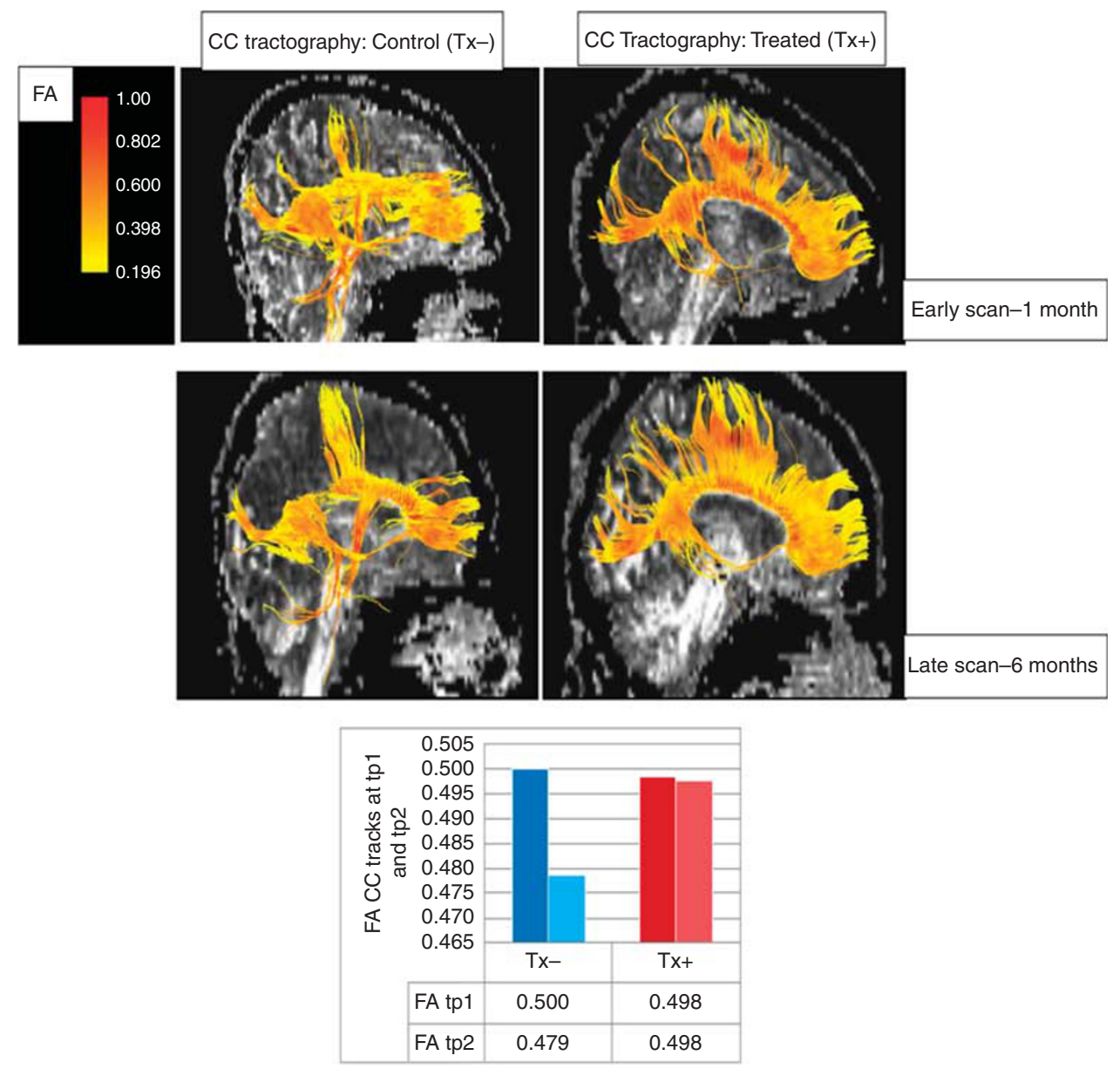

Figure 3. Central nervous system (CNS) structural preservation with bone marrow mononuclear cell (BMMNC). Since our phase I trial in children, we have focused on structural preservation as a viable surrogate outcome in traumatic brain injury (TBI). This figure shows the diffusion tensor imaging magnetic resonance imaging (DT MRI) focus on the corpus callosum, and the volumetric measurement as well as the fractional anisotropy (FA) and the underlying table (Table 1) shows how these measures correlate with neurocognitive outcomes. These data were presented in Stem Cells(38).

concept driving the translation of the approach in terms of dosing regimen.

Adult phase I/IIa trial of BMMNC for severe TBI. Using the same cell type and dosing paradigm, 25 patients were enrolled in a trial evaluating safety and potential structural preservation of CNS after severe TBI (38). The adult study confirmed the logistical feasibility and safety of the autologous approach, and demonstrated a treatment effect in terms of structural preservation of key regions of interest (corpus callosum, corticospinal tract) that correlate with neurocognitive outcomes. Further, there was a dampening of the proinflammatory cytokine response to injury. These data provided/confirmed a treatment effect size based upon structural preservation outcome measures of $\sim 50$ patients. There was no difference in the Glasgow Outcome Scores between groups.

Follow-on studies: Currently there are two (adults and children, separately) phase IIb trials using a Bayesian adaptive design comparing two doses of BMMNC vs. controls in a prospective, double-blind, randomized trial that uses imaging end points as the putative biomarker for efficacy. Specifically, there is a global hypothesis that by reducing the neuroinflammatory response to injury that the typical $8-12 \%$ total volumetric loss noted after severe TBI at 6-12 months after injury will be attenuated. As of this writing, the pediatric trial has enrolled 33 of the planned 50 total number of patients. The adult trial has enrolled 11/55 planned patients as of this writing.

Chronic TBI (SanBio). SanBio is sponsoring a clinical trial to treat patients with chronic TBI and isolated motor deficits using sterotactically implanted, genetically modified MSCs. A handful of patients have been enrolled as of the time of this writing, and no interim results are available. These cells are being implanted at perilesional sites with a presumed paracrine mechanism of action. They have a similar trial in chronic stroke and have demonstrated mixed results, depending upon the outcome measure considered.

\section{Spinal Cord Injury}

OPCs (Geron-Asterias: SCiStar study). The SCiStar trial seeks to test three sequential escalating doses of AST-OPC-1 up to 20 million total cells in as many as 35 patients with 

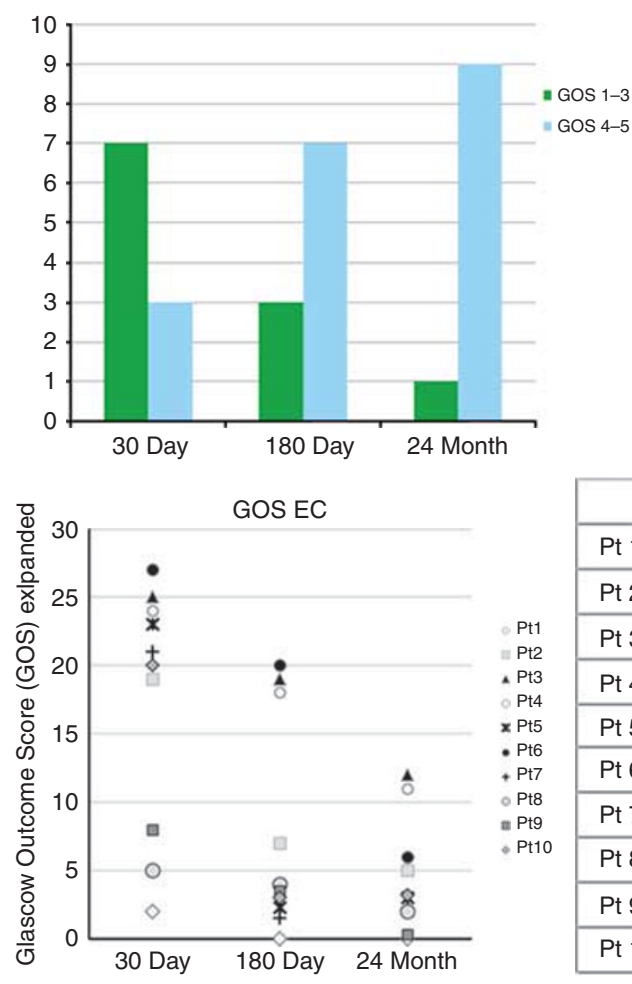

\begin{tabular}{|c|c|c|c|}
\hline & 30 Day & 180 Day & 24 Month \\
\hline Pt 1 & 2 & 0 & 0 \\
\hline $\mathrm{Pt} 2$ & 19 & 7 & 5 \\
\hline $\mathrm{Pt} 3$ & 25 & 19 & 12 \\
\hline $\mathrm{Pt} 4$ & 24 & 18 & 11 \\
\hline Pt 5 & 23 & 2.3 & 3 \\
\hline $\mathrm{Pt} 6$ & 27 & 20 & 6 \\
\hline $\mathrm{Pt} 7$ & 21 & 1.5 & 2.2 \\
\hline Pt 8 & 5 & 4 & 2 \\
\hline Pt 9 & 8 & 3.5 & 0.3 \\
\hline Pt 10 & 20 & 3 & 3.2 \\
\hline
\end{tabular}

Figure 4. Evidence of progressive improvement in functional outcomes in children after 6 months, and these data were originally published there without the longer term follow-up data(33) Conventionally, the Glasgow Outcome Score (GOS) is measured at 6 months as there is little probability of changing a category after that time(42). In this phase I trial, there was a 70:30\% dichotomized good:bad outcome at 6 months, which was consistent with the literature in severe traumatic brain injury (TBI) in children. However, at 2 years after injury, there was continued improvement such that the dichotomized GOS was 90:10\% good:bad. In one of the few trials with 2-year long-term GOS data in children with severe TBI, the Thomale study(43)showed a 73\%:27\% good:bad outcome, without progressive improvement. This phenomenon of progressive improvement past standard time windows was also observed in the MASTERS trial in adult, ischemic stroke using multipotent adult progenitor cells infused intravenously. They observed a progressive, statistically, and clinically significant improvement in the treatment group relative to controls at 1 year relative to the traditional 90 -day end point in stroke trials(44).

subacute C5-C7, motor complete cervical SCI. The direct injection will be at 14-30 days after injury and the patients will be followed by neurological exams and imaging followup. AST-OPC-1 is an ESC-derived oligodendrocyte precursor cell line that has reparative function that focuses on remyelination of demyelinated axons in the spinal cord. In preclinical testing, the remyelination was associated with improved extremity function and reduction in the size of the injury cavity. In a previous phase I trial, 2 million cells were administered in the thoracic position at 7-14 days after injury accompanied by immunosuppression for 60 days. There were no concerning safety events, but the study was not designed to evaluate for efficacy-and importantly, none was seen. This has been attributed to a dosing issue, as 2 million cells is a small number that was felt to have a lower probability of success in terms of efficacy. The 10 million cell dose cohort has not been reported outside of press releases (Asteriasbiotherapeutics.com/inv_news_releases.php), but they have noted a one-level motor improvement in all six patients at 6 months, without safety concerns.

Fetal brain-derived NSCs (Pathway Trial-Stem Cells). Recently, Stem Cells halted a clinical trial (A single-blind, randomized, parallel arm, Phase 2 Proof of Concept Study of the safety and efficacy of Human Central Nervous System (HuCNS-SC) Transplantation in Cervical Spinal Cord Injury) for presumed futility. Although there was a press release of initial modest clinical improvements, the effect was not durable, and may have been associated with the planned withdrawal of the immunosuppression theoretically resulting in graft loss. This trial sought to determine the efficacy of intramedullary transplantation of fetal-derived NSCs into the cervical spinal cord in patients with $\mathrm{C} 5-\mathrm{C} 7$ motor levels with American Spinal Injury Association Impairment Scale Grades $\mathrm{B}$ or C at 12 weeks after injury. They excluded patients with complete cord transection. A recent series of publications raised significant questions regarding the translation of the preclinical data $(32,39-41)$. The reports focused questions on either the poor predictive value of the animal models or a loss of potency in the cell product when translated into cyclic guanosine monophosphate production of the clinical grade cells for the trial. Serious questions arose after the publication by the Anderson lab that could not replicate any benefit of the clinical grade cells as used in the trial. Importantly, there was some improvement in the animals treated with the research grade cell line; however, even though the results with the research grade cells were positive, they were not compelling. 


\section{Cell therapy for TBI/SCI Review}

Table 1. Correlation of fractional anisotropy of corpus callosum region with outcomes, $r(P)$

\begin{tabular}{|c|c|c|c|c|c|c|}
\hline Outcomes & CC1 & $\mathrm{CC} 2$ & $\mathrm{CC} 3$ & CC4 & CC5 & Whole CC \\
\hline \multicolumn{7}{|l|}{ Functional } \\
\hline Glasgow Outcome Scal—Extended ${ }^{a}$ & $0.238(0.299)$ & $0.300(0.187)$ & $0.196(0.394)$ & $0.373(0.096)$ & $0.747(0.000)$ & $0.583(0.006)$ \\
\hline $\begin{array}{l}\text { Mayo-Portland Adaptability Index- } \\
\text { Composite }\end{array}$ & $-0.238(0.298)$ & $-0.120(0.603)$ & $-0.133(0.565)$ & $-0.212(0.356)$ & $-0.461(0.036)$ & $-0.381(0.088)$ \\
\hline \multicolumn{7}{|l|}{ Motor and Processing Speed } \\
\hline Grooved Pegboard-Dominant Hand ${ }^{a}$ & $0.330(0.145)$ & $0.655(0.001)$ & $0.679(0.001)$ & $0.509(0.018)$ & $0.637(0.002)$ & $0.761(0.000)$ \\
\hline Trail Making Test $\mathrm{B}^{\mathrm{a}}$ & $0.253(0.268)$ & $0.512(0.018)$ & $0.520(0.016)$ & $0.488(0.025)$ & $0.594(0.005)$ & $0.687(0.001)$ \\
\hline WAIS Processing Speed Index & $0.168(0.468)$ & $0.679(0.001)$ & $0.771(0.000)$ & $0.611(0.003)$ & $0.692(0.001)$ & $0.767(0.000)$ \\
\hline $\begin{array}{l}\text { Rey Auditory Verbal Learning Test- } \\
\text { Delayed Recall }\end{array}$ & $0.320(0.157)$ & $0.488(0.025)$ & $0.504(0.020)$ & $0.482(0.027)$ & $0.414(0.062)$ & $0.572(0.007)$ \\
\hline \multicolumn{7}{|l|}{ Verbal fluency } \\
\hline Controlled Oral Word Association Test ${ }^{a}$ & $0.205(0.374)$ & $0.575(0.006)$ & $0.558(0.009)$ & $0.321(0.155)$ & $0.260(0.255)$ & $0.475(0.030)$ \\
\hline
\end{tabular}

Bolded values significant at $P<0.05$

aspearman's $\rho$.

Further complicating the issue, there was the assertion in the publication that warnings from the preclinical team were not considered fully during the translation process. Specifically, there was a claim that the clinical cell line did not meet the predescribed milestones for efficacy, and these data were communicated (but not acted upon) with the company. The trial went forward after consideration of those data, ultimately failing to demonstrate durable efficacy. This issue highlights a few critical issues in translating cellular therapies for neurological injuries. First, although the animal models are instructive, they do not necessarily have a strong positive or negative predictive value in terms of translation into a clinical outcome-an issue rigorously described in Narayan's overview published in 2002 (2). This has been well established in the TBI literature, and it is made more problematic when using immunologically deficient animals to enhance engraftment. Second, clinical Good Manufacturing Practice processing of a cell product may require alteration of particular reagents or processes that may change the ultimate cell product potency. Third, cell identity assays that function as a surrogate for potency are inadequate, especially when relying upon engraftment as a mechanism of action.

\section{CONCLUSIONS}

Since the early proof of concept data that is now over 20 years old, there has been progressive movement of cell-based therapies to treat neurological injuries-including translation into pediatric patients. The early data point to hints of efficacy, but clearly there are not miraculous "cures" noted in these phase I-II trials. The future will undoubtedly build upon these early results to refine the cellular products (focusing on potency and cell types) and identify the dosing regimens that will further improve upon this early foundation of work.

\section{ACKNOWLEDGMENTS}

The Program in Pediatric Regenerative Medicine is indebted to gracious and generous donors, Clare A. Glassell and Evelyn P. Griffin.

\section{STATEMENT OF FINANCIAL INTEREST}

The work described in this article was supported by NIH/NINDS 5RO1 NS077963-05, DOD Joint Warfighter Program W81XWH-16-C-0040, State of Texas Emerging Technology Fund, and Bentsen Center for Stroke Research.

Disclosures: $\operatorname{Dr}$ Cox has equity and royalty interests in Cellvation, Sponsored Research related to this publication from National Institutes of Health, Department of Defense, Cellvation, Athersys, Celgene Cellular Therapeutics, and CBR.

\section{REFERENCES}

1. Kraus JF, McArthur DL. Epidemiological aspects of brain injury. Neurol Clin 1996;14:436-50.

2. Narayan R, Michel ME, Ansell B, et al. Clinical trials in head injury. J Neurotrauma 2002;19:503-57.

3. Maramou A. A review of progress in understanding the pathophysiology of brain edema. Neurosurg Focus 2007;22:E1.

4. Ghajar J. Traumatic brain injury. Lancet 2000;356:923-9.

5. Luersson TG. Neurological injuries in infants and children: an overview of current management strategies. Clin Neurosurg 2001;12:170-84.

6. Adelson PD, Bratton SL, Carney NA, et al. Guidelines for the medical management of severe acute traumatic brain injury in infants, children 
and adolescents: recommendations from the traumatic brain injury clinical trials network. J Head Trauma Rehabil 2010;25:375-82.

7. Bratton SL, Chestnut RM, Ghajar J, the Brain Trauma Foundation: AANS/CNS Joint Section on Neurotrauma and Critical Care. Guidelines for the management of severe traumatic brain injury: I-XV. J Neurotrauma 2007;24 (Suppl): 1-95.

8. Robertson CS, Vladka AB, Hannay HJ, et al. Prevention of secondary ischemic insults after severe head injury. Crit Care Med 1999;27:2086-95.

9. Downard C, Hulka F, Mullins RJ, et al. Relationship of cerebral perfusion pressure and survival in pediatric brain injured patients. J Trauma 2000;49:654-9.

10. Hutchinson JS, Ward RE, Lacroix J, et al. Hypothermia after traumatic brain injury in children. N Eng J Med 2008;358:2447-56.

11. Jackson M, Srivastava A, Cox CS. Pre-clinical progenitor cell therapy in traumatic brain injury: a meta-analysis. J Surg Res 2017;214:38-48.

12. Harting MT, Jimenez F, Xue H, et al. Intravenous mesenchymal stem cells for traumatic brain injury? J Neurosurg 2009;110:1189-97.

13. Coyne TM, Marcus AJ, Woodbury D, Black I. Marrow stromal cells transplanted to the adult brain are rejected by an inflammatory response and transfer donor labels to host neurons and glia. Stem Cells 2006;24:2483-92.

14. Walker PA, Bedi SS, Shah SK, et al. Intravenous multipotent adult progenitor cell therapy after traumatic brain injury: modulation of the resident microglia population. J Neuroinflamm 2012;9:228-41.

15. Bedi SS, Walker PA, Shah SK, et al. Autologous bone marrow mononuclear cell therapy attenuates activated microglia/macrophage response and improves spatial learning after traumatic brain injury. J Trauma Acute Care Surg 2013;75:410-6.

16. Walker PA, Shah SK, Jimenez F, et al. Intravenous multipotent adult progenitor cell therapy for traumatic brain injury: preserving the blood brain barrier via an interaction with splenocytes. Exp Neurol 2010;225: 341-52.

17. Lu D, Sanberg PR, Mahmood A, et al. Intravenous administration of human umbilical cord blood reduces neurological deficit in the rat after traumatic brain injury. Cell Transplant 2002;11:275-81.

18. Savitz SI, Cox CS. Cell therapies for stroke and traumatic brain injury: targeting microglia. Stem Cells 2016;34:537-42.

19. Savitz SI, Misra V, Kasam M, et al. Intravenous autologous bone marrow mononuclear cells for ischemic stroke. Ann Neurol 2011;70:59-69.

20. Hocum Stone LL, Xiao F, Rotschafer J, et al. Amelioration of ischemic brain injury in rats with human umbilical cord blood stem cells: mechanisms of action. Cell Transplant 2016;25:1473-88.

21. Hentze H, Soong PL, Wang ST, et al. Teratoma formation by human embryonic stem cells: evaluation of essential parameters for future safety studies. Stem Cell Res 2009;2:198-210.

22. Menge T, Zhao Y, Zhao J, et al. Mesenchymal stem cells regulate bloodbrain barrier integrity through TIMP3 release after traumatic brain injury. Sci Transl Med 2012;4:161-7.

23. Watanabe J, Shetty AK, Hattiangady B, et al. Administration of TSG-6 improves memory after traumatic brain injury in mice. Neurobiol Dis 2013;59:86-99.

24. Zhang Y, Chopp M, Meng, et al. Effect of exosomes derived from multipluripotent mesenchymal stromal cells on functional recovery an neurovascular plasticity in rats after traumatic brain injury. J Neurosurg 2015;122:856-67.

25. Pati S, Khakoo A, Zhao J, et al. Human mesenchymal stem cells inhibit vascular permeability by modulating VE-cadherin/B-catenin signaling. Stem Cells Dev 2011;20:89-101.
26. Bedi SS, Hetz R, Thomas C, et al. Intravenous multipotent adult progenitor cell therapy improves spatial learning after traumatic brain injury. Stem Cells Transl Med 2013;2:953-60.

27. Mahmood A, Lu D, Li Y, et al. Intracranial bone marrow transplantation after traumatic brain injury improving functional outcome in adult rats. J Neurosurg 2001;94:589-95.

28. Mahmood A, Lu D, Qu C, Goussev A, Chopp M. Long-term recovery after bone marrow stromal cell treatment of traumatic brain injury in rats. J Neurosurg 2006;104:272-7.

29. Harting MT, Sloan LE, Jimenez F, Baumgartner J, Cox CS. Subacute neural stem cell therapy for traumatic brain injury. J Surg Res 2009;153: 188-94.

30. Keirstead HS, Nistor G, Bernal G, et al. Human embryonic stem cellderived oligodendrocyte progenitor cell transplants re-myelinate and restore locomotion after spinal cord injury. J Neurosci 2005;25:4694-705.

31. Cao Q, He Q, Wang Y, et al. Transplantation of ciliary neurotropic factorexpressing adult oligodendrocyte precursor cells promotes re-myelination and functional recovery after spinal cord injury. J Neurosci 2010;30: 2989-3001.

32. Anderson AJ, Piltti KM, Hooshmand MJ, Nishi RA, Cummings BJ. Preclinical efficacy failure of human CNS-derived stem cells for use in the Pathway study of cervical spinal cord injury. Stem Cell Rep 2017;8:249-63.

33. Cox CS, Baumgartner JE, Harting MT, et al. Autologous bone marrow mononuclear cells for severe traumatic brain injury in children. Neurosurgery 2015;68:588-600.

34. Sidaros A, Skimminge A, Liptrot MG, et al. Long term global and regional brain volume changes following severe traumatic brain injury: a longitudinal study with clinical correlates. Neuroimage 2009;44:1-8.

35. Wilde EA, Hunter JV, Newsome MR, et al. Frontal and temporal morphometric findings on MRI in children after moderate to severe traumatic brain injury. J Neurotrauma 2005;22:333-44.

36. Liao GP, Harting MT, Hetz RA, et al. Autologous bone marrow mononuclear cells reduce therapeutic intensity for severe traumatic brain injury in children. Pediatr Crit Care Med 2015;16:245-55.

37. Guiza F, Depreitere B, Piper B, et al. Visualizing the pressure and time burden of intracranial hypertension in adult and pediatric traumatic brain injury. Intens Care Med 2015;41:1067-76.

38. Cox CS, Hetz RA, Liao GP, et al. Treatment of severe adult traumatic brain injury using bone marrow mononuclear cells. Stem Cells 2016;35: 1065-79.

39. Temple S, Studer L. Lessons learned from pioneering neural stem cell studies. Stem Cell Rep 2017;8:191-3.

40. Monuki ES, Anderson AJ, Blurton-Jones M, Cummings BJ. Response to Stem Cells Inc. Stem Cell Rep 2017;8:195-7.

41. Stem Cells Inc. former management. Reaction from Stem Cells Inc. to two papers in Stem Cell Reports on the efficacy of human NSCs in mouse models of Alzheimer's disease and spinal cord injury. Stem Cell Rep 2017;8:194-5.

42. Teasdale GM, Pettigrew LWL, Wilson JTL, Murray G, Jennett B. Analyzing outcome of treatment of severe head injury: a review and update on advancing the use of the Glasgow Outcome Scale. J Neurotrauma 1998;15:587-96.

43. Thomale UW, Graetz D, Vajkoczy, Sarrafzadeh AS. Severe traumatic brain injury in children: a single center experience regarding therapy and long-term outcome. Childs Nerv Syst 2010;26:1563-73.

44. Hess DC, Wechsler LR, Clark WM, et al. Safety and efficacy of multipotent adult progenitor cells in acute ischaemic stroke (MASTERS): a randomized, double-blind, placebo controlled, Phase 2 trial. Lancet Neurol 2017;16:320-68. 